\section{Procesos de pensamiento: ¿Cómo DESARROLLARLOS A TRAVÉS DE LA INVESTIGACIÓN EN EL MARCO DE ENSEÑANZA PARA LA COMPRENSIÓN (EPC)?}

\author{
THOUGHT PROCESSES: \\ HOW TO DEVELOP THEM THROUGH RESEARCH \\ WITHIN THE FRAMEWORK OF TEACHING FOR \\ UNDERSTANDING (EPC)?
}

María Paola Bermúdez Vásquez*
* Magíster en Educación de la
Universidad Surcolombiana.
Docente de ciclo I ( $1^{\circ}, 2^{\circ}$
y $3^{\circ}$ de básica primaria)
de la Institución Educativa
Colombus American School
del sector privado.
mpaola0521@yahoo.es
0000-0001-9784-8013

Cómo citar este artículo: Bermúdez, V. M. P. (2021). Procesos de Pensamiento: ¿cómo desarrollarlos a través de la investigación en el marco de enseñanza para la comprensión (Epc)?. Revista PACA 11, pp. 91-105.
Aceptado: Octubre 4, 2021

Resumen: En la Institución Educativa Colombus American School, del municipio de Rivera, Huila, se realizó el proyecto "Desarrollo de los procesos de pensamiento en niños de ciclo $1\left(1^{\circ}, 2^{\circ}\right.$ y $3^{\circ}$ de básica primaria) a través de la investigación en el marco de la enseñanza para la comprensión", mediante un enfoque cualitativo de tipo etnográfico, con el objetivo de valorar el desarrollo de los procesos de pensamiento a través de la investigación como mediación pedagógica y didáctica básica en niños en el marco de la enseñanza para la comprensión.

En este contexto se considera importante identificar que la investigación dentro de la escuela o ámbito escolar permitiría la potencialización de todas las capacidades y habilidades desarrollando seres competentes, críticos y constructores de escenarios propicios para el desarrollo humano en sus diferentes dimensiones. Considerando que, desde la implementación de estrategias investigativas, los estudiantes y docentes pueden alcanzar niveles de desempeño superiores, no solo para el aula sino también formación para la vida.

Teniendo esto en cuenta, toma mayor fuerza la inquietud de los educadores por formar y fomentar una 
escuela diferente. Es allí donde la investigación como mediadora pedagógica y didáctica se convierte en la piedra angular de la formación educativa y del desarrollo de los procesos de pensamiento, permitiendo fortalecer el desarrollo de la crítica, lógica y la creatividad.

Palabras clave: cultura investigativa, procesos de pensamiento, prácticas pedagógicas, Enseñanza para la comprensión (Epc), secuencia didáctica.

Abstract: In the Colombus American School Educational Institution, in the municipality of Rivera, Huila, the project "development of thinking processes in children of cycle I (1st, 2nd and 3rd grade of elementary school) through research in the framework of teaching for understanding" was carried out through a qualitative ethnographic approach, with the objective of assessing the development of thinking processes through research as a basic pedagogical and didactic mediation in children in the framework of teaching for understanding.

In this context, it is considered important to identify that research within the school or school environment would allow the potentiation of all capacities and abilities, developing competent, critical beings and builders of favorable scenarios for human development in its different dimensions. Considering that, from the implementation of research strategies, students and teachers can reach higher levels of performance not only for the classroom but also for life.

With this in mind, the concern of educators to form and promote a different school becomes stronger. It is there, where research as a pedagogical and didactic mediator becomes the cornerstone of educational training and the development of thought processes allowing to strengthen the development of criticism, logic and creativity.

Keywords: research culture, thinking processes, pedagogic practices, Teaching for Understanding, didactic sequence.

\section{Introducción}

Las instituciones educativas en todos sus niveles escolares tienen el compromiso y la responsabilidad de formar en sus estudiantes la comprensión y la transformación de sus conocimientos en aprendizajes significativos. La construcción del conocimiento y la producción del nuevo saber en educación y pedagogía se plantea desde la construcción de nuevos aprendizajes autónomos, colaborativos y significativo y la necesidad de innovación en educación y pedagogía desde el pensamiento divergente 
de los educadores-mediadores, con ingenio, creatividad, capacidad de invención y disposición prospectiva a la transformación.

Es a la luz de esta perspectiva que el desarrollo del pensamiento a través de la investigación como mediación pedagógica y didáctica ofrece nuevas alternativas para la interpretación de las realidades siempre aceptadas, y el ser humano moderniza su forma de ser, de sentir, de actuar y de pensar. Sin embargo, la escuela se ha ido quedando rezagada y fragmentada frente a estos desarrollos y por esta razón las prácticas de aula exigen un acoplamiento con los procesos de aprendizaje de los individuos, potenciando la capacidad de pensamiento, en lugar de transmitir información, compilar datos y fechas.

En paralelo con las ideas anteriormente expuestas, la práctica docente ha de ser redefinida, repensada y enfocada al desafío de la comprensión y el pensamiento, partiendo de transformaciones voluntarias producto del desarrollo actitudinal, del convencimiento y de la vivencia de la necesidad del cambio. Esto, indiscutiblemente hace que sea necesario establecer otra forma, manera o herramienta pedagógica que facilite el alcance de estos propósitos. Es allí donde se identifica y se reconoce que la investigación dentro de la escuela o ámbito escolar potencializa todas las capacidades y habilidades para desarrollar seres competentes, críticos y constructores de escenarios propicios para el desarrollo humano en sus diferentes dimensiones.

En tal sentido, en esta investigación se parte de la hipótesis de que los estudiantes de ciclo I conformado por los grados primero, segundo y tercero de la básica primaria de la Institución Educativa Colombus American School de Rivera - Huila, se involucran de forma activa en su propio aprendizaje. Por lo tanto, se expondrá un planteamiento del problema con los factores determinantes de la misma, describiendo un marco referencial exponiendo. De igual forma, se abordan unos antecedentes, la metodología, la caracterización de la población seleccionada y, finalmente, las conclusiones y recomendaciones obtenidas de la investigación.

\section{Referentes teóricos}

En primer lugar, se indican algunos estudios relacionados, partiendo del concepto de mayor relevancia que es el de la cultura de la investigación. 
De acuerdo con García Vigil 202, s.f. se describe la cultura de Investigación como la práctica institucionalizada que configura a cada uno de los actores académicos para resolver de forma científica los problemas propios de su área de especialidad, hacer uso de las competencias con que cuentan en cultura de Información, cultura de conocimiento y cultura de comunicación; dicha práctica es una práctica especializada en la generación de nueva información y conocimientos a partir de la instrumentación de tales competencias. La cultura de investigación es necesaria, pues a través de la misma los actores académicos incorporarán una manera de producir y manejar información, de entender, interpretar, explicar y comunicar los resultados de una investigación desde una perspectiva científica que les permitirá operar más allá del sentido común.

Así mismo, la investigación en educación se fundamenta en la Ley 115 de 1994, Ley General de Educación y sus decretos reglamentarios; de este modo, la política educativa en Colombia incorpora los procesos investigativos en las instituciones educativas, asumiéndolos como una actividad humana inherente a la práctica pedagógica, la cual posibilita la construcción de conocimiento y saberes complejos a través de espacios de reflexión crítica, colaboración, participación e innovación.

Además, los fines del Plan Decenal de Educación 2006-2016 disponen:

\begin{abstract}
"La educación como política de Estado debe materializarse en políticas, planes, programas, proyectos y acciones que promuevan la cultura, la investigación, la innovación, el conocimiento, la ciencia, la tecnología y la técnica, que contribuyan al desarrollo humano integral, sostenible y sustentable, a través de la ampliación de las oportunidades de progreso de los individuos, las comunidades, las regiones y la nación" $(2005$, p. 5)
\end{abstract}

Por otro parte, el Programa Ondas define la investigación como "una actividad propia del ser humano, posible de desarrollar en todas las áreas del conocimiento y con niños y niñas muy pequeñas, también como un proceso de desciframiento de la condición humana a partir de la experiencia de vida de esta población en los contextos escolares, familiares y comunitarios" (Mejía, 2015)

En esa misma línea, plantea Hernández (2014) que la cultura investigativa, así como el espíritu investigativo, son características naturales en los seres humanos. El deseo de averiguar el porqué y el cómo 
de las cosas que nos rodean es algo tan innato que lleva a las personas a indagar y cuestionar; por tanto, no puede creerse que los estudiantes carezcan de la cultura investigativa, más bien es falta de desarrollarla.

Aunado a esto, desde el punto de vista de Chamorro (2008), para crear el espíritu investigativo es necesario fomentar la imaginación, la búsqueda de la verdad, la curiosidad inalcanzable, el hábito de la lectura, la motivación. Para entender el desarrollo científico e investigativo debemos tener en claro la importancia de la investigación, considerando que esta ha sido concebida como el proceso para descubrir nuevos conocimientos, en donde se puede implementar un espíritu investigativo que se expresa y descubre mediante la curiosidad, el ensayo y el error, formulándose una pregunta inteligente, para posteriormente resolverla mediante un proceso, en la inclinación y aptitud de la razón humana que sirve de fundamento a nuestros actos, para procurar descubrir lo que se ignora.

En tal sentido, la investigación en la escuela se ha ido consolidando como el eje transformador del currículo desde un enfoque de pedagogías activas (Montessori, 2003), que fortalece la aplicación de las inteligencias múltiples (Gardner, 1994) como dinamizador de los planes de estudio y facilitador de las interacciones maestro - estudiante para generar una contextualización del aprendizaje y el redescubrimiento del conocimiento. Todo lo anterior es posible a través de la observación y la indagación que el maestro orienta en el aula para facilitar un aprendizaje significativo que sea utilizado por y para la vida.

Por último, para la construcción del diseño investigativo se vincula el Proyecto Zero que se basa en la investigación y propuestas sobre aprendizaje, razonamiento e inteligencia. Uno de los programas de investigación propuestos por el Proyecto Zero, son los postulados de Jerome Bruner (1960), Nelson Goodman (1967), Howard Gardner David Perskin (1972) y Carlos Eduardo Vasco (2009). De allí nace el proyecto de Enseñanza para la Comprensión (EpC) con los referentes de Tyna Blythe (2009), Patricia León y Ximena Barrera (1996), que se fundamenta en que el estudiante pueda ir más allá de lo aprendido, haciendo uso del conocimiento en situaciones nuevas para resolver problemas, generando procesos de pensamiento donde explique con sus propias palabras, ejemplifique las situaciones, realice experimentos para corroborar sus aprendizajes e identifique las relaciones con el medio y con la vida cotidiana. 
PROCESOS DE PENSAMIENTO: ¿Cómo deSARROLlaRLos A TRAVÉS DE LA INVESTIGACIÓN EN EL MARCO...

\title{
Metodología
}

La investigación es de naturaleza etnográfica:

\begin{abstract}
"La investigación etnográfica es el método más popular para analizar y enfatizar las cuestiones descriptivas e interpretativas de un ámbito sociocultural concreto. Ha sido ampliamente utilizada en los estudios de la antropología social y la educación, tanto que puede ser considerada como uno de los métodos de investigación más relevantes dentro de la investigación humanístico-interpretativa" ((Latorre, del Rinón, \& Arnal, 2005)).
\end{abstract}

El enfoque metodológico de la presente investigación responde a la metodología cualitativa, orientada a explorar, describir, interpretar y comprender las didácticas existentes que promuevan el desarrollo de la investigación como una forma de pensar, sentir y de actuar en los niños del ciclo I de Educación Básica Primaria del Colombus American School, ubicado en el municipio de Rivera, Huila, a partir de un análisis documental del plan de estudios institucional, el cual se ha visto fortalecido a través de la implementación de una nueva asignatura que promueve la investigación escolar y posterior acercamiento a las prácticas pedagógicas que implementan los docentes en el proceso enseñanza / aprendizaje, con el fin de verificar su aplicabilidad y operatividad en concordancia con el enfoque pedagógico y las últimas adecuaciones curriculares institucionales.

La población objeto de estudio se enmarcó dentro de la institución educativa Colombus American School, la cual se encuentra ubicada geográficamente en la vereda Arenoso del municipio de Rivera, zona sur del Huila. Para la investigación se tuvo una muestra poblacional de 25 niños (Ver Tabla No. 1. Distribución de la población estudiantil Colombus American School); de igual forma se involucran 3 docentes, distribuidos en las áreas de lengua castellana, ciencias naturales, ciencias sociales, y 9 padres de familia.

En ese mismo contexto, el trabajo de campo permite integrar información procedente de fuentes y contextos diversos; por ello, los momentos o fases de la investigación no constituyen una secuencia lineal rígida; antes bien se caracteriza por el paso a paso, en una profundización de manera progresiva. Los momentos o fases de la investigación se desarrollaron de la siguiente manera: 


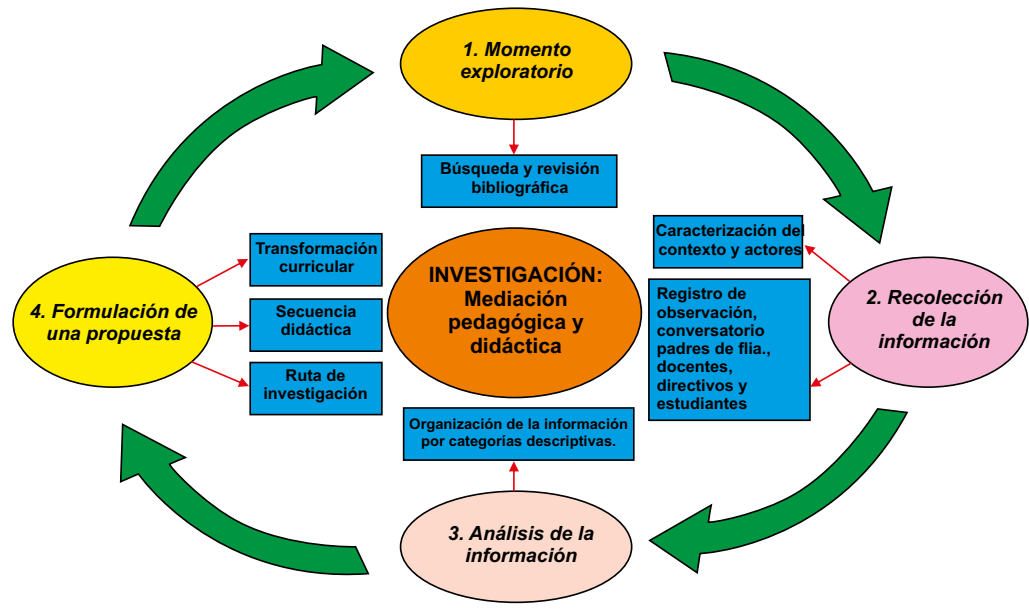

Figura 1. Momentos de la investigación de desarrollo de procesos de pensamiento a través de la investigación en el marco de la Epc. Construcción gráfica para la realización de la presente investigación.

Primer momento: Exploratorio: se emprende la búsqueda y la revisión de fuentes bibliográficas de los antecedentes, con el propósito de conocer de manera global acerca de los avances de la investigación como mediación pedagógica y didáctica en el desarrollo de procesos de pensamiento en los procesos de enseñanza en el aula; análisis documental desde la perspectiva del enfoque pedagógico institucional, vinculando una desestructuración curricular donde el docente y el estudiante se conciban como investigadores promoviendo la búsqueda, la indagación y la divulgación de hallazgos, incentivando el desarrollo de procesos de pensamiento como elemento fundamental.

Segundo momento: Recolección de la información: en esta etapa se tendrán en cuenta la caracterización de los contextos y de los actores, la recolección de la información a través de la aplicación de la técnicas e instrumentos dialógicos (Registro de observación, discusión dirigida y registro de campo).

Tercer momento: Análisis de la información: una vez realizada la organización de la información por categorías descriptivas arrojadas de los interrogantes y objetivos del estudio, se procede al análisis inductivo mediante la búsqueda de patrones comunes en los datos obtenidos con los docentes, directivos y padres de familia, objeto de este estudio, la construcción de tendencias y relaciones entre categorías. 
PROCESOS DE PENSAMIENTO: ¿Cómo deSARROLlaRLos A TRAVÉS DE LA INVESTIGACIÓN EN EL MARCO...

Cuarto momento: Formulación de una propuesta: este momento inicialmente estará formado por el análisis de relación entre los objetivos y problema de investigación con los resultados obtenidos y el análisis a los datos realizado. Por ello se hace necesario que posteriormente se proceda a establecer las líneas de acción para la valoración de la investigación como mediación pedagógica y didáctica básica para el desarrollo de procesos de pensamiento en niños del ciclo I de educación básica primaria.

\section{Resultados}

En el análisis de los resultados, en primer lugar se realiza el diagnóstico inicial a través del formato de observación de prácticas pedagógicas en el aula, aplicado mediante la técnica de observación participante activa o directa, lo cual permite la observación de clases en un ambiente real con los diferentes docentes y estudiantes del nivel de básica primaria de ciclo I. En cada una de las observaciones de las prácticas de aula se evidencian tres fases: la primera, explorando saberes; seguidamente, investigación guiada; y, por último, producción de síntesis.

En la primera fase, explorando saberes, se hacen visible los saberes previos o preconceptos que tienen los estudiantes referentes al objeto de estudio. En este caso, los estudiantes lograron experimentar actividades dinámicas que impulsaron sus preguntas hacia nuevos conocimientos de forma espontánea, permitiendo un proceso de metacognición más evidente a través de experiencias significativas y dinámicas con un enfoque científico, vivenciando diferentes situaciones problematizadoras, registrando en sus bitácoras todo lo que ellos observaban, sus preguntas de sentido común en problemas de investigación; luego realizaron la categorización de sus preguntas pensando en qué era lo que les inquietaba y como complemento se realiza la categorización de sus problemas de investigación, detallando la frecuencia con la que se relacionaban sus aportes y así finalizar con un solo planteamiento del problema.

La fase de investigación guiada permite contextualizar el problema enseñando a buscar diferentes alternativas para rastrear fuentes confiables, ofreciendo herramientas para procesar información y caracterizar el problema planteado, identificando conceptos asociados. Aquí es importante destacar que los estudiantes se centran en la idea de ¿quiénes se han hecho la misma pregunta problematizadora? Teniendo 
como recursos las consultas, la documentación, representaciones gráficas, videos e invitados especiales. En esta fase dieron respuesta a sus preguntas problematizadoras.

En los aspectos relacionados con la fase de producción de síntesis de la observación de las prácticas de aula es conveniente decir que se hace énfasis en la verificación del proceso de comprensión de los estudiantes sobre el problema a investigar, realizando análisis de la información, planteando posibles soluciones y divulgando los hallazgos.

En lo que atañe a la reflexión que realizan los padres de familia, es notorio que coinciden en que investigar es un proceso que permite la construcción de conocimiento personal o colectivo que implica preguntar, consultar, indagar y que es esencial que esa búsqueda sea intencionada o provocada.

Por lo que se refiere a la investigación en el aula la ven como un conjunto de métodos a través de un proceso ordenado, consciente y didáctico. Para ellos, es muy importante que la investigación en el aula motive a niños y niñas a buscar interrogantes aproximándolos a que tengan pensamientos divergentes.

A partir de las ideas anteriores, los padres de familia consideran que la planeación curricular está favoreciendo la investigación, porque es una herramienta pedagógica donde el conocimiento se adquiere de forma crítica partiendo de su aplicación transversal, abarcando diversas áreas, partiendo de preguntas y obteniendo resultados concretos. Pero a su vez, es muy importante el papel del docente porque es el que debe lograr motivar a los estudiantes para que sea posible enseñarles a investigar acerca de sus curiosidades, aunque hace falta más comprensión, alineación y hasta comunicación entre los diferentes actores (profesores, directivos, padres de familia) que rodean y guían al estudiante.

Por último, es preciso señalar que la audiencia foco expresa que el modelo institucional propicia el desarrollo del espíritu investigativo, por una parte, porque los estudiantes han aprendido a argumentar sus ideas y opiniones con más coherencia, seguridad y tranquilidad; asimismo, entendieron que existen diferentes formas de organizar la abstracción de la información, determinando los conceptos claves sienten que esto 
PROCESOS DE PENSAMIENTO: ¿Cómo deSARROLLARLOS A TRAVÉS DE LA INVESTIGACIÓN EN EL MARCO...

les facilita la vida de tal manera que les permite solucionar cualquier situación problematizadora.

\section{Conclusiones}

A partir del proceso de investigación realizado se concluye que describir los procesos de pensamiento que surgen de las prácticas de aula priorizan la investigación como mediación pedagógica y didáctica; cabe destacar que el desarrollo de procesos de pensamiento inicia con enseñar a pensar sobre lo que cada uno hace y descubrir que tiene un talento que se autodesconocía. De ahí que ser necesario que el educador registre y documente cada fase del aprendizaje del educando para identificar la estructura mental que tiene cada uno de ellos.

Así mismo, en esta investigación se pudo evidenciar que los procesos de pensamiento se desarrollan evolutivamente de acuerdo con la complejidad del objeto de estudio, asociando la edad y la madurez mental de los estudiantes. En este orden de ideas, el pensamiento es considerado un proceso interno que se hace visible a través de la práctica de cuestionar, escuchar y documentar, convirtiéndose en algo concreto y real; se torna en una estructura sobre la que se puede hablar, explorar, manipular, desafiar y aprender.

En las prácticas pedagógicas se dio prioridad a la investigación y se hicieron visibles los siguientes procesos de pensamiento:

- Proceso de pensamiento mecánico: La noción de este proceso de pensamiento se centra en codificar y consolidar información o datos. Las operaciones mentales que los estudiantes desarrollaron fueron recolectar información, procesar información, almacenar información, retener información, recordar información y evocar información.

- Proceso de pensamiento concreto: Este proceso de pensamiento se basa en lo que se ve y lo que se asimila centrándose en el mundo físico y sus elementos y, a su vez, la realidad externa. Para esto se vale de operaciones mentales como, en primera medida, partir de las experiencias vividas para elaborar imágenes mentales y organizar ideas, elaborar conceptos y tomar postura crítica frente a ellos.

- Proceso de pensamiento configurativo: Por lo que se refiere a este proceso de pensamiento, el estudiante adopta una postura 
haciendo una apuesta a la multidiversidad, multidisciplinariedad y a la multidimensionalidad, teniendo como punto de partida la realidad propia y no la externa.

- Proceso de pensamiento de abstracción: Este proceso demanda las siguientes operaciones mentales: describir el objeto de abstracción, determinar lo esencial del objeto y despreciar los rangos y nexos secundarios y no determinantes del objeto, explicar, concluir y argumentar.

Por otra parte, se ubica al docente como mediador del proceso de aprendizaje desarrollado por el estudiante, es decir, que el docente desde el rol que cumple es decisivo, pues implica hacerse responsable de la manera como los estudiantes relacionan sus expectativas hacia el conocimiento y el descubrimiento. El docente- mediador debe concebirse como un ser privilegiado en el proceso educativo, pues de él depende el éxito que los estudiantes alcancen su madurez intelectual, a través de la investigación en el aula, aplicable a cualquier área del conocimiento; el hombre, como ser social por naturaleza, se hace -o rehace- en la medida en que se deja influir por el sistema educativo y sus procesos de transformación de su entorno.

A su vez, es importante precisar que la exploración, la investigación y la producción de síntesis conforman la estructura fundamental, la cual hace recorrer los procesos de pensamiento desarrollados en los estudiantes, destacando los avances significativos. Por consiguiente, la didáctica de la exploración, la investigación y la producción de síntesis permite un pensar y actuar flexible, con lo que el estudiante sabe y con los nuevos conocimientos, situaciones que le facilitan ir más allá de un pensamiento y acción memorística y rutinaria, facilitándole la construcción de su propio conocimiento, entendiendo el porqué y para qué aprender.

Finalmente, la exploración, la investigación y la producción de síntesis son determinantes en la comprensión, puesto que realizan un proceso interactivo, en el cual los educandos, aparte de construir una representación organizada y coherente del aprendizaje, provoca en el estudiante estimulación del desarrollo de pensamiento, llevándolo a la reflexión, más allá de las imágenes mentales, de modo que satisfaga sus necesidades de una manera autónoma, contribuyendo al fortalecimiento del espíritu investigativo. 
PROCESOS DE PENSAMIENTO: ¿Cómo deSARROLLARLOS A TRAVÉS DE LA INVESTIGACIÓN EN EL MARCO...

\section{Referencias Bibliográficas}

Acosta, A. Estrategias pedagógicas para fomentar competencias investigativas en las docentes de ciencias naturales del Colegio Hijas de Cristo Rey. Universidad de la Sabana. 2011.

Briones, G. (2003). Métodos y técnicas de la investigación. México: Trillas.

Blythe, T. (1999). Enseñanza para la comprensión, Guía docente. Buenos Aires: Paidós.

Ceniceros, D. (2003). El profesor como investigador. INED No. 2. Universidad Pedagógica de Durango.

Cerda, A. M. (2004). El complejo camino de la formación ciudadana: una mirada a las prácticas docentes. Lom ediciones. Fabra, M. L. (1994). Técnicas de grupo para la Cooperación. Grupo Planeta (GBS).

Colombia, M. d. (s.f.). La práctica pedagógica como escenario de aprendizaje. Obtenido de:https://www.mineducacion.gov.co/1759/articles-357388_ recurso_1.pdf

Contreras, A. G., \& Ladino Ospina, Y. (2008). Desarrollo de competencias científicas a través de una estrategia de enseñanza y aprendizaje por investigación. Bogotá: Studiositas.

Costamagna, A. y Manuale, M. Estrategias de enseñanza para la comprensión: un enfoque alternativo. Recuperado de: http:// gimnasiocampestrelosarrayanesedu.com/assets/epc-info-base.pdf

102 Chamorro, H. (1 de Febrero de 2008). Por la cultura ciéntifica e investigativa. Obtenido de: http://trejochamorro.blogspot.com/2008/02/algunasorientaciones-para-un-docente.html

Dewey, J. (1982). Cómo pensamos. Barcelona: Paidós.

Escribano, A. (2010). El aprendizaje basado en problemas. Bogotá, Colombia: Narcea

Enseñar a pensar, Nuevo currículum: Project Zero. (2015) Recuperado de https:// observatorio.profuturo.education/blog/2015/01/08/ensenar-a-pensarnuevo- curriculum-project-zero/

Fernández, S. P. (septiembre de 1997). La Teoría Crítica de la Sociedad Legado y Diferencias en Teoría de la Comunicación Cinta de Moebio No. 1. Chile. Recuperado de: http://rehue.csociales.uchile.cl/publicaciones/moebio/01/ frames30.htm

García, Vigil 202. (s.f.). Universidad Mesoamericana. Obtenido de: Universidad Mesoamericana: http://www.lameso.edu.mx/oaxaca/investigacion/culturade-investigacion/

García, D. (2015) Tesis: Rutinas de pensamiento, una estrategia para desarrollar el pensamiento y la comprensión en los niños de preescolar. Universidad de la Sabana. Chía, Cundinamarca, Colombia.

Gardner, H. (1994). Enseñar para la comprensión en las disciplinas - y más allá de ellas. Teachers College Record, Volume 96, Number 2. 
Gardner, H. (1997). Estructuras de la mente. La teoría de las inteligencias múltiples. México. Fondo de Cultura Económica.

González, F. L. (2000). Investigación Cualitativa en Psicología. Rumbos y desafíos. México: S.A. Ediciones Parninfo.

Grupo de investigación para el desarrollo social y tecnológico. (Enero de 2014). Recuperado de Grupo de investigación para el desarrollo social y tecnológico: https://scienti.colciencias.gov.co/gruplac/jsp/visualiza/ visualizagr.jsp?nro $=00000000015149$

Harvard. (s.f.). Project zero. Recuperado el 17 de 10 de 2013 de Visible thinking http://www.pz.gse.harvard.edu/index.php

Hernández, R. (2014). Metodología de la investigación. México: McGrawHill Educatión.

Hernández, M. (1980). La relación entre pensamiento y lenguaje según Piaget, Vygotsky, Luria y Bruner, Anales de la Universidad de Murcia.

Howard, G. y Boix-Mansilla, V. Enseñar para la comprensión en las disciplinas y más allá de ellas. (1994). Traducción al español Lion, C. Teachers College Record Volumen 96, Number 2.

Ianfranceco, G. (2012). Aprendizaje Autónomo y Cognición. Bogotá: Coripet.

Jaramillo, A. A., \& Pupo Jaramillo, L. R. (2011). Estrategias pedagógicas para fomentar competencias inestigativas en las docentes de ciencias naturales del Colegio las Hijas de Cristo Rey. Bogotá.

Latorre, A., del Rinón, D., \& Arnal, J. (2005). Bases metodológicas de la investigación educativa. Barcelona: Ediciones Experiencia.

León, P y Barrera, X. Ruta maestra, Ed. 9. (2013). Recuperado de: http:// fundacies.org/site/wp-content/uploads/2013/01/EpC-Ruta.pdf)

León, P y Barrera, X. Adaptación del libro: La Enseñanza para la Comprensión. Vinculación entre la investigación y la práctica. (1999). Buenos Aires. Editorial Paidós.

López, N. E. (2017). Currículo crítico o estandarizado, un debate necesario. Educación y Cultura, 60.

Matute, E. (27 de Noviembre de 2019). Instituto de Neurociencias. Obtenido de Instituto de Neurociencias: http://ineuro.cucba.udg.mx/articulo.php?id=9

Mejía, M. M.-M. (julio de 2015). Estrategia No. 3: Autoformación, formación colaborativa, producción de saber y conocimientoy apropiación para maestros apoyada en NTCI. Proyecto; Fortalecimiento de la apropiación social de la ciencia, la tecnología y la innovación apoyados en NTCI en el departamento de Santander. Bucaramanga, Colombia: Fractus. Recuperado de http://fractussantander.fitec.la/repodoc/frontend/files/get/estrategia_3_ autoformacin_de_maestro/Estrategia-3-autoformacion-colaborativaproduccion-de-saber-y-conocimiento-y-aprobacion-para-maestrosaproyada-en-NTIC.pdf 
PROCESOS DE PENSAMIENTO: ¿Cómo deSARROLLARLOS A TRAVÉS DE LA INVESTIGACIÓN EN EL MARCO...

Mejía, M. (2019). Departamento de Administración de Ciencia, Tecnología e Innovación - Colciencias. Recuperado de https://www.colciencias.gov.co/ cultura-en-ctei/ondas

Mejía, M. Praxis \& saber, vol. 2. 2011. Investigación como estrategia pedagógica, una apuesta por construir pedagogías críticas en el siglo XXI. P. 127. Recuperado de: https://revistas.uptc.edu.co/revistas /index.php/praxis_ saber/article/view/1127

Ministerio de Educación Nacional. (s.f.). Recuperado de: https://www. mineducacion.gov.co/1621/article-80185.html

Ministerio de Educación Nacional. (s.f.). Recuperado de https://www. mineducacion.gov.co/1621/article-80185.html

Montessori, M. (2003). El método de la pedagogía cientifica aplicado a la educación de la infancia. Madrid: Biblioteca Nueva.

Molano, M. (2011). Carlos Eduardo Vasco Uribe. Trayectoria biográfica de un intelectual colombiano: una mirada a las reformas curriculares en el país. Revista Colombiana de Educación, 61. Bogotá, Colombia.

Nacional, M. d. (2009). Desarrollo infantil y competencias de la primera infancia. Bogotá.

Perkins, D. (s.f.). La escuela inteligente. Gedisa.

Novak, J. (1988). Aprendiendo a aprender. Martínez Roca, Barcelona.

Nussbaum, M. (2001). Paisajes del pensamiento. Barcelona, Paidós.

104 Pardo Romero, S. L., Arévalo, L. M., \& Quiazua, M. Y. (2014). Desarrollo del pensamiento crítico a partir de rutinas de pesamiento. Chía.

Pérez, D. G., Marcedo, B., Sifredo, C., Valdés , P., \& Viches, A. (2005). ¿Cómo promover el interés por la cultura científica? Una propuesta didáctica fundamentada para la educación científica de jóvenes de 15 a 18 años. Chile: Unesco.

Pérez, M. (2009). La aparición del pensamiento. Una enmienda emotiva a la tesis de Donald Davidson, Granada, UGR.

Perskin, D. (1990). El marco de la enseñanza para la comprensión. Boston, Massachusetts.

Perskin, D. (2003). Pensamiento visible. Paidós. Recuperado de: http://formacion. intef.es/pluginfile.php $/ 48457 / \mathrm{mod}$ imscp/content $/ 1 /$ pensamiento_invisible. html

Piaget, J. (1987). El lenguaje y el pensamiento del niño pequeño. Barcelona: Paidós.

Poject Zero. (8 de Enero de 2015). Recuperado de: https://observatorio.profuturo. education/blog/2015/01/08/ensenar-a-pensar-nuevo-curriculum-projectzero/

Pujadas, B. (2016). Construyendo saber etnográfico: reflexiones a partir de la experiencia de campo en instituciones escolares. Athenea Digital.

Quintanilla, M. R. (2013). Desarrollo de habilidades y competencias de pensamiento científico en estudiantes y profesores y su relación con la 
adquisición del conocimiento pedagógico del contenido para enseñar en High School. Chile.

Ritchhart, R., \& Perskin, D. (2014). Hacer visible el pensamiento. En R. Ritchhart, Cómo promover el compromiso, la comprensión y la autonomía de los estudiantes. Buenos Aires: Paidós. Voces de la educación.

Rosa, M. J. (2009). Habilidades básicas del pensamiento y competencia. Nuevas estrategias, nuevos aprendizajes.

Salmon, A. (2008). Promoting a Culture of thinking in the young child. Lectura y Vida.

Sánchez, M. (2002). La investigación sobre el desarrollo y la enseñanza de las habilidades de pensamiento. Revista Electrónica de Investigación Educativa 4, (1). Recuperado el 24 de Junio de 2013 de http://dialnet.unirioja.es/ servlet/articulo?codigo $=239480$

Stone, M (1999). Enseñanza para la Comprensión "Vinculación entre la investigación y la práctica”. Buenos Aires. Editorial Paidós.

Tishman, S., \& Palmer, P. (2005). Pensamiento Visible. Obtenido de https:// educrea.cl/wp-content/uploads/2017/01/DOC2-estrategias-pens-visible.pdf

Vasco, C. E. (2001). Pedagogías para la comprensión en las disciplinas académicas. Uniplur/iversidad, vol. 1, No. 3.

Vigotsky, L. (1964). Pensamiento y lenguaje. Buenos Aires: Lautaro.

Wilson, D. Proyecto Zero: el pensamiento es algo que se puede aprender. (2017). Recuperado de http://www.revistadeeducacion.cl/daniel-wilson-d i r e c t or proyecto-zero-pensamiento-algo-se-puede-aprender/

Zilberstein, J. (2009). Aprendizaje y enseñanza en una educación por ciclos. Bogotá. Editorial Magisterio. 